\title{
2-Deoxy-glucose Enhances the Effect of Cisplatin and Pemetrexed in Reducing Malignant Pleural Mesothelioma Cell Proliferation But Not Spheroid Growth
}

\author{
IRINI GEROGIANNI ${ }^{1}$, ELEANNA PITARAKI ${ }^{2}$, RAJESH M. JAGIRDAR ${ }^{2}$, OLYMPIA KOULIOU ${ }^{2}$, \\ LYDIA GIANNAKOU ${ }^{2}$, STEFANOS GIANNOPOULOS ${ }^{2}$, ELEFTHERIOS PAPAZOGLOU ${ }^{2}$, \\ CHRISSI HATZOGLOU ${ }^{1,2}$, KONSTANTINOS I. GOURGOULIANIS ${ }^{1}$ and SOTIRIOS G. ZAROGIANNIS ${ }^{1,2}$ \\ ${ }^{1}$ Department of Respiratory Medicine, Faculty of Medicine, University of Thessaly, BIOPOLIS, Larissa, Greece; \\ ${ }^{2}$ Department of Physiology, Faculty of Medicine, University of Thessaly, BIOPOLIS, Larissa, Greece
}

\begin{abstract}
Background/Aim: Malignant pleural mesothelioma $(M P M)$ is a therapy-resistant neoplasm of the pleura. Standard chemotherapy consists of a combination of cisplatin $(C P D D)$ and pemetrexed (PEM). The aim of this study was to assess whether inhibition of aerobic glycolysis by 2-deoxyglucose $(2 D G)$ would enhance the effects of standard chemotherapy. Materials and Methods: MeT-5A, M14K, MSTO and ZL34 cell lines were used. Cell viability with $2 D G$ and cell proliferation and spheroid formation with $C P D D+P E M$ alone and with 2-DG were tested. Results: Viability with 2-DG was dose-dependent. Cell proliferation with $C P D D+P E M$ on $2 D$ surface was reduced in all cell types, 2-DG inclusion demonstrated a synergistic effect in MSTO and ZL34 cells. Spheroid growth in $3 D$ with $C P D D+P E M$ or $C P D D+P E M+2-D G$ lowered spheroid growth in all cell types. Conclusion: 2-DG synergizes with $C P D D+P E M$ in lowering MPM cell proliferation in $2 D$ to $<20 \%$. In 3D MPM spheroid growth 2-DG synergism with $C P D D+P E M$ treatment is not maintained.
\end{abstract}

Malignant pleural mesothelioma (MPM) is a therapyresistant neoplasm of the pleura arising from asbestos exposure with a median survival after diagnosis of around one year (1). In a cohort of 19,000 MPM cases it was shown that with no treatment, the overall survival was 4.8 months,

Correspondence to: Sotirios G. Zarogiannis, Ph.D., M.P.H., Environmental Pleural \& Lung Diseases Group, Department of Physiology, Faculty of Medicine, University of Thessaly, 3 Panepistimiou Str., BIOPOLIS, Larissa 41500, Greece. Tel: +30 2410685558, Fax: +30 2410685555, e-mail: szarog@med.uth.gr

Key Words: 2-deoxy-glucose, 2D cell cultures, 3D cell cultures, cell proliferation, chemotherapy, malignant pleural mesothelioma, Warburg effect. while with chemotherapy the overall survival was reported to be 11.3 months (2). The most frequent histotype of MPM is epithelioid followed by biphasic and sarcomatoid. The sarcomatoid histotype is considered to be the most aggressive followed by the biphasic and epithelioid type (3). Usually due to late diagnosis the tumor is unresectable, therefore the main treatment is chemotherapy by the combination of cisplatin (CPDD) and pemetrexed (PEM) (1).

A characteristic feature of MPM compared to other solid tumors, is that it develops as a sheet in the pleural membrane (in a 2-dimentional like fashion) wrapping and congesting the lung parenchyma (4). On the other hand, the spontaneous formation of spheroid structures in explant culture indicates an inherent property of the primary cells to recapitulate a morphological 3-dimentional character, that may be associated with local dissemination of MPM in the pleural cavity. Previously, pleural fluid cytology demonstrated the presence of 3-dimentional spheroid like cell aggregates with coiled cords of cells, small papillae with a central core and at times tissue fragments with pseudoacinar formation (5). Therefore, when assessing the efficacy of chemotherapeutic agents in vitro, it is reasonable to do so both in 2-D and 3-D cultures.

CPDD and PEM treatment have been shown to directly induce the expression of multidrug resistance $\mathrm{ABC}$ transporter proteins like ABCG2 in biphasic and sarcomatoid mesothelioma cell models. The induction of such transporters results in the expulsion of chemotherapeutic agents that renders MPM chemo-resistant (6). Furthermore, cell-extracellular matrix (ECM) interactions apart from determining tumor cell implantation to novel sites, also induce the expression of multidrug resistance $\mathrm{ABC}$ transporter proteins like $\mathrm{ABCC} 1$, contributing to chemoresistance of the tumor (7). Thus, ECM, an acellular component of the tumor structure influences the MPM pathophysiology. 
In order to counteract chemo-resistance, some investigators have attempted to manipulate the tendency of cancer cells to depend on aerobic glycolysis for their energy production (Warburg effect), which is also the case in MPM cells. It was shown that citrate in combination with cisplatin was effective in enhancing the cisplatin cytotoxic effects in vitro in MSTO cells, a chemo-resistant biphasic MPM cell line (8). Furthermore, the same group tested this approach in vivo in a model of intraperitoneal carcinomatosis using MSTO cells and 3bromopyruvate (3-BrPA), as the Warburg effects inhibiting factor in combination to cisplatin (9). In this setting it was shown that although cisplatin alone had mild effects in improving mice survival, the addition of 3-BrPA significantly enhanced the survival of mice. This points to the importance of glycolysis for the viability of MPM cells and hence tumor growth.

In line with the above, the aim of the current study was to administer 2-DG in combination to first-line chemotherapy (CPDD+PEM) in benign mesothelial cells and in epithelioid, biphasic and sarcomatoid cell lines in order to assess their effects on 2-D and 3-D cell growth. This approach would provide information regarding the potential of this treatment in reducing the 2D tumor growth encountered in MPM development and the 3D spheroid mediated local metastasis within the pleural cavity.

\section{Materials and Methods}

Cell culture and drugs. Cells used in this study were MeT-5A (benign mesothelial cells), M14K (epithelioid MPM), MSTO-211H (MSTO, biphasic MPM) and ZL34 (sarcomatoid MPM). Cells were cultured in $10 \%$ FBS in RPMI with L-Glutamine and antibiotics (complete media; Sigma, Darmstadt, Germany). For synchronization purposes, cells were maintained in $0.5 \%$ FBS-RPMI for $24 \mathrm{~h}$.

Drugs. 2-Deoxy-glucose, (2-DG, D8375-1G, Sigma), cisplatin (CPDD, PHR1624-200MG, Sigma) and pemetrexed (PEM, CDS024404-50MG, Sigma) stock solutions were diluted into complete media and used as indicated.

ECM treatment of culture surface. ECM treatments were performed as described previously (10). Cells were seeded with complete media in 96 -well plates $\left(10^{4}\right.$ cells/well), and cultured for $48 \mathrm{~h}$. The cells were then dissolved with sterile ECM extraction buffer $(0.5 \%$ Triton $\times 100,1 \%$ sodium deoxycholate in PBS) followed by $2 \times$ washes with sterile PBS and a treatment with DNaseI for $30 \mathrm{~min}$. The wells were then washed again once with sterile PBS and immediately used for experiments.

Colorimetric assay with crystal violet stain. Cells were washed with PBS followed by fixation with $4 \%$ para-formaldehyde followed by staining with $0.5 \%$ crystal violet $(\mathrm{CV})$ stain. The cells were then gently washed 3 times with tap water to remove unbound stain, followed by drying overnight. The CV stain was released with $10 \%$ acetic acid and measured at $540 \mathrm{~nm}$ using a spectrophotometer.

Cell viability assay. Cells $\left(5 \times 10^{3} /\right.$ well) were seeded on ECM treated plates and maintained in $0.5 \%$ FBS RPMI media for $24 \mathrm{~h}$. Viability of cells in 2-dexoy-glucose at concentrations of $0.5 \mathrm{mM}, 1.0 \mathrm{mM}$, $2.0 \mathrm{mM}$ and $3.0 \mathrm{mM}$ were tested for $48 \mathrm{~h}$ in complete media. At the end of the assay, each plate was subject to colorimetric assay. All experiments were repeated twice with an $n=8$ in each experiment.

Cell proliferation assay. Serum starved, synchronized cells $\left(5 \times 10^{3} /\right.$ well $)$ were seeded on ECM treated plates with complete media (Control), with complete media containing CPDD $(10 \mu \mathrm{M})$ + PEM $(200 \mu \mathrm{M})$, or the combination of $2 \mathrm{DG}(3.0 \mathrm{mM})$ with CPDD+PEM. Media were changed after $48 \mathrm{~h}$. At the end of the $72 \mathrm{~h}$, plates were subjected to colorimetric assay. Each experiment was repeated twice with an $n=4-6$ in each experiment.

Spheroid formation assay. Serum-starved, synchronized cells ( 50 cells in $25 \mu \mathrm{l}$ ) were cultured in a hanging drop configuration for $72 \mathrm{~h}$ in complete media (Control) or along with CPDD+PEM or $2 \mathrm{DG}+\mathrm{CPDD}+\mathrm{PEM}$. All treatments received $250 \mathrm{ng} / \mathrm{ml}$ homologous ECM. The spheroids were imaged for perimeter measurement with ImageJ. All experiments were repeated twice with an $n=8-12$ in each experiment.

Statistical analysis. Analyses were performed using Prism 8 for Mac. Data were tested for normality with D'Agostino \& Pearson omnibus normality test and comparisons were performed with Oneway ANOVA and Dunnett or Tukey multiple-comparisons post-hoc test where appropriate for parametric data, while for non-parametric data comparisons were made with Kruskal-Wallis and Dunn's multiple-comparisons post-hoc test. All data are presented as means \pm SEM. Values of $p<0.05$ were deemed significant.

\section{Results}

Benign mesothelial and MPM cells demonstrate dose-dependent viability with 2-dexoy-glucose. All cell types demonstrated significant reduction in viability with increasing $2 \mathrm{DG}$ doses of $0.5 \mathrm{mM}, 1.0 \mathrm{mM}, 2.0 \mathrm{mM}$ and $3.0 \mathrm{mM}$. 2DG at $3.0 \mathrm{mM}$ dose in MeT-5A cells (Figure 1A) significantly reduced viability (Control: 100.0 $\pm 5.6 \%, 2 \mathrm{DG}: 45.0 \pm 4.4 \%, p<0.001$ ). In M14K cells (Figure 1B), viability was significantly reduced compared to control (Control: $100.0 \pm 7.4 \%, 2 \mathrm{DG}: 47.0 \pm 2.5 \%, p<0.001$ ). In MSTO cells (Figure 1C) the viability was significantly reduced compared to control (Control: $100.0 \pm 6.8 \%, 2 \mathrm{DG}$ : $36.0 \pm 1.2 \%, p<0.0010$ ), and in ZL34 cells (Figure 1D) the viability was significantly reduced compared to control (Control: 100.0 $\pm 8.5 \%, 2$ DG:50.9 $\pm 6.5 \%, p<0.001$ ).

Biphasic and sarcomatoid MPM cells show higher susceptibility to 2-DG+CPDD+PEM compared to CPDD+ PEM treatment on $2 D$ surface. In MeT-5A cells, mean \% cell proliferation was significantly lower in cell media with drugs compared to control (Control: 100 $\pm 5.0 \%$, CPDD+PEM: $23.0 \pm 4.0 \%, 2 \mathrm{DG}+\mathrm{CPDD}+\mathrm{PEM}: 10.6 \pm 0.6 \% ; p<0.001$ versus Control; Figure 2A). In M14K cells, in media with drugs the mean $\%$ cell proliferation was significantly lower compared to control (Control: $100 \pm 1.6 \%$, CPDD+ PEM: $13.0 \pm 0.8 \%$, $2 \mathrm{DG}+\mathrm{CPDD}+\mathrm{PEM}: 11.2 \pm 0.5 ; p<0.001$ versus Control; Figure 2B). In MSTO cells, mean \% cell proliferation in media with 

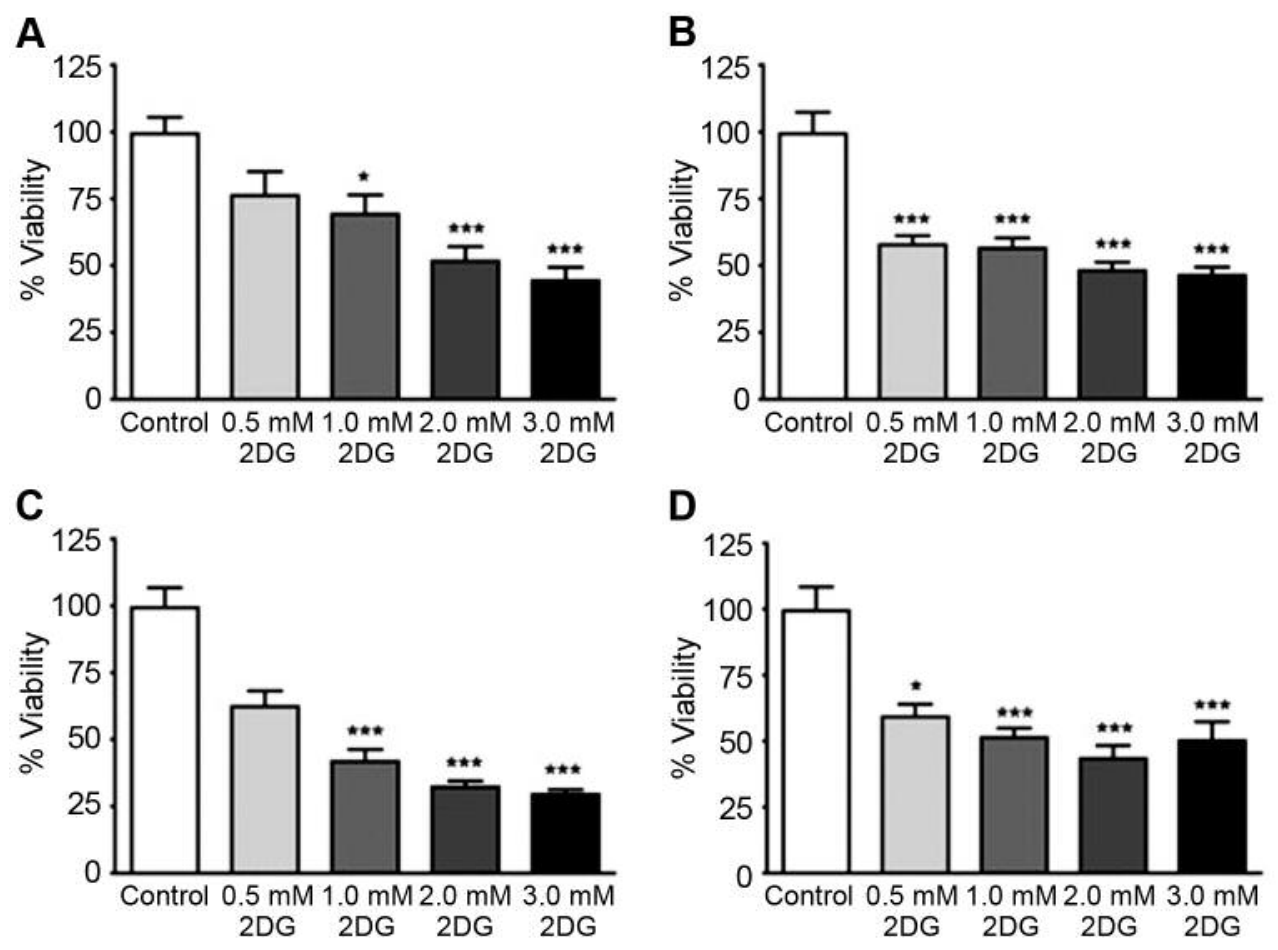

Figure 1. Mean \pm SEM \% viability of (A) MeT-5A cells, (B) M14K cells, (C) MSTO cells and (D) ZL34 cells. *p<0.05 and ***p<0.001 versus Control. Data combined from 2 independent experiments, $n=8$ in each experiment.

drugs was significantly lower compared to control (Control: $98.7 \pm 2.5 \%$, CPDD+PEM: $16.0 \pm 0.7 \%, 2 \mathrm{DG}+\mathrm{CPDD}+\mathrm{PEM}$ : $5.4 \pm 0.1 \% ; p<0.001$ versus Control; Figure $2 \mathrm{C})$. Additionally, the mean $\%$ cell proliferation in $2 \mathrm{DG}+\mathrm{CPDD}+\mathrm{PEM}$ was significantly lower $(p<0.001)$ compared to CPDD+PEM treatment (Figure 2C). In ZL34 cells, mean \% cell proliferation in media with drugs was significantly lower compared to

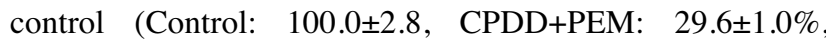
2DG+CPDD+PEM: $12.5 \pm 0.7 ; p<0.001$ versus Control; Figure $2 \mathrm{D})$. Also, the mean $\%$ cell proliferation in $2 \mathrm{DG}+\mathrm{CPDD}+\mathrm{PEM}$ was significantly lower $(p<0.001)$ compared to CPDD+PEM treatment (Figure 2D).

$3 D$ spheroid cultures are equally sensitive to $C P D D+P E M$ and $2 D G+C P D D+P E M$. In 3D spheroids of MeT-5A cells, drug treated spheroids had a significantly lower $\%$ mean perimeter compared to control (Control: $100.0 \pm 3.6 \%$, CPDD+ PEM: $68.9 \pm 1.4 \%, 2 \mathrm{DG}+\mathrm{CPDD}+\mathrm{PEM}$ : $69.5 \pm 2.3 \% ; p<0.001$ versus Control; Figure $3 \mathrm{~A}$ ). In $\mathrm{M} 14 \mathrm{~K}$ cells (Figure $3 \mathrm{~B}$ ), the treated $\%$ mean perimeter of spheroid was significantly lower compared to control (Control: 100.0 $2.0 \%$, CPDD+PEM: $67.0 \pm 1.6 \%, 2 \mathrm{DG}+\mathrm{CPDD}+\mathrm{PEM}: 69.6 \pm 2.3 \% ; p<0.001$ versus Control). In MSTO cells (Figure 3C), the treated \% mean perimeter of spheroid was significantly lower compared to control (Control: 100.0 $\pm 2.2 \%$, CPDD+PEM: 78.3 $\pm 1.7 \%$,
2DG+CPDD+PEM: $77.0 \pm 1.8 \% ; p<0.001)$. Finally, in ZL34 cells (Figure 3D), the $\%$ mean perimeter of treated spheroids was significantly lower compared to control (Control:

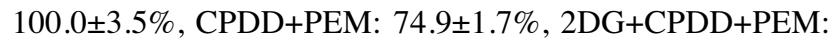
$72.5 \pm 1.1 \% ; p<0.001$ versus Control).

\section{Discussion}

In this study we have shown that treatment of benign mesothelial and malignant mesothelioma cells renders them susceptible to inhibition of glycolysis by 2-DG treatment. The epithelioid and sarcomatoid histological subtypes (M14K and ZL34 cell lines respectively) were more sensitive to 2-DG treatment since they had significantly decreased viability even with $0.5 \mathrm{mM}$ treatment, while all cell lines had a viability on the range of $50 \%$ when treated with $3 \mathrm{mM}$ of 2-DG. Compared to other cell types, mesothelial cells were more susceptible to 2-DG than the NSCLC cell lines A549 and PC-9 as well as the liver cancer cell lines HepF2 and Hep3B, but more resilient than papillary thyroid carcinoma cells CG3 (11-13). Contrary to the above experiments, the experiment we performed in this study used homologous cell derived ECM as a substrate, as described previously (10). This approach provides an additional layer of information given that cell-ECM interaction has been reported to 

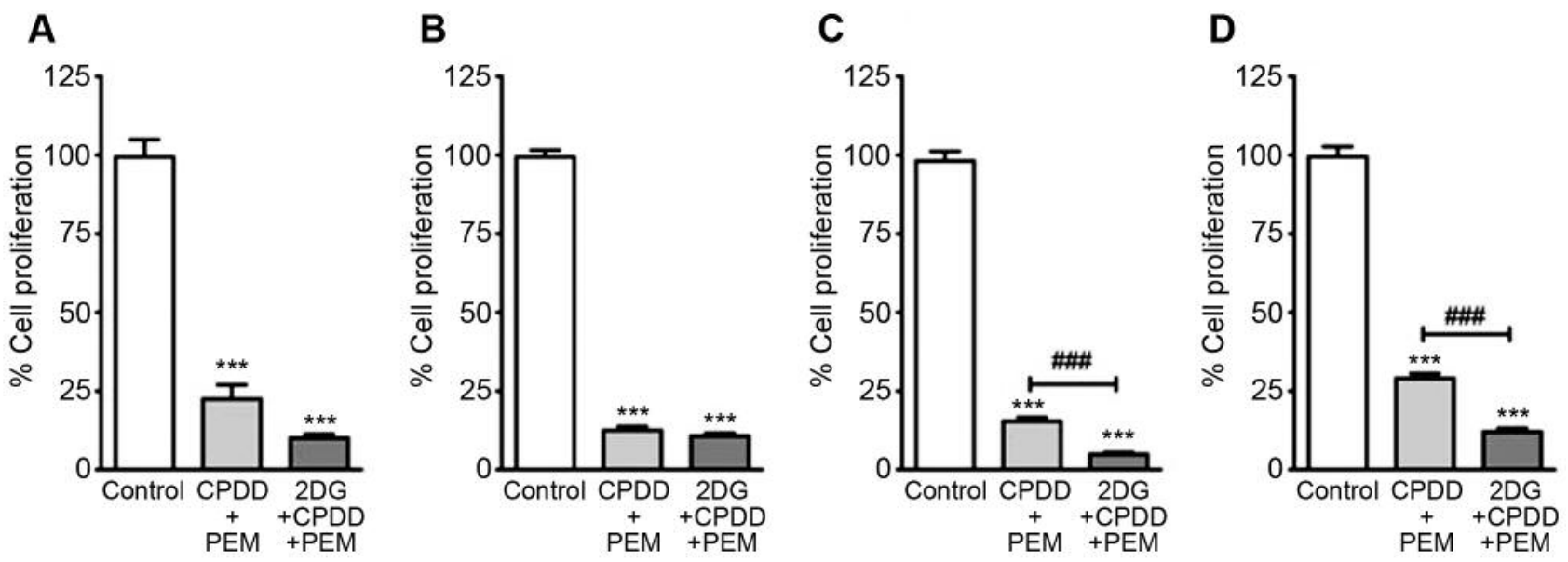

Figure 2. Mean \pm SEM \% cell proliferation of (A) MeT-5A cells after 72 h, (B) of M14K cells, (C) of MSTO and (D) of ZL34. ***p<0.001 versus Control, ${ }^{\# \#} p<0.001$ versus $C P D D+P E M$. Data combined from 2 independent experiments, $n=4-6$ in each experiment.
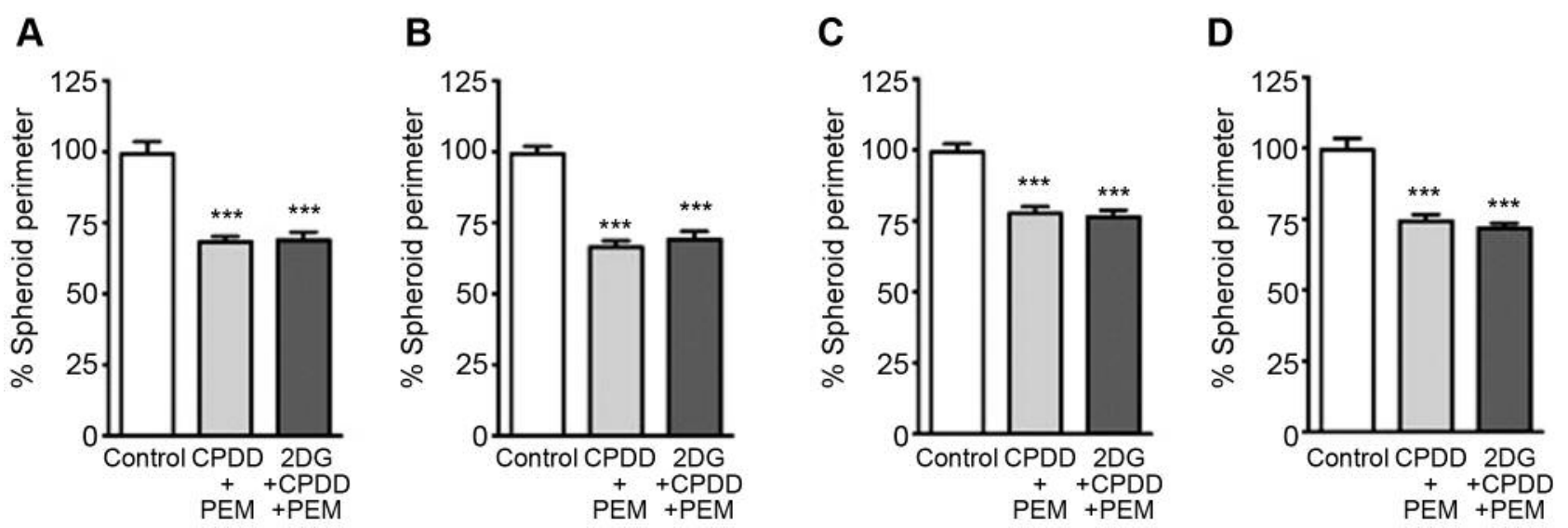

Figure 3. Mean \pm SEM \% spheroid perimeter after 72 h of (A) MeT-5A cells, (B) of M14K cells, (C) of MSTO and (D) of ZL34. ***p<0.001 versus Control. Data combined from 2 independent experiments, $n=8-12$ in each experiment.

contribute to the development of chemoresistance in tumors (7). Our in vitro system recapitulates well the cell-ECM interactions encountered in vivo.

Based on the above findings we also assessed whether 2-DG would enhance the effects of the first-line chemotherapeutics, CPDD and PEM, in decreasing tumor cell growth in 2D and $3 \mathrm{D}$, testing namely cell proliferation and spheroid formation phenotypes. In both cases the homologous cell derived ECM component was maintained. 2DG in combination with CPDD and PEM showed $<25 \%$ proliferation in all cell types. In MSTO and ZL34 cells, 2DG synergized with CPDD+PEM treatment, which is suggestive of dependence on glucose metabolism. The 3D spheroid formation assay differs from cell proliferation in $2 \mathrm{D}$ in that, the cells are subject to ECM without a solid substratum. Spheroid formation and growth limits drug availability in the interior of the spheroid structure. Our data demonstrate $75 \%$ growth under cytotoxic challenge that is in contrast to 25-30\% growth on 2D surface. In MPM, this is the first study demonstrating experimental data on 2D and 3D cell line models with $2 \mathrm{DG}$ as a therapeutic and with homologous cell derived ECM. The spontaneous formation of spheroid structures in explant cultures indicates an inherent property of primary cells to recapitulate a morphological character associated with their neoplastic nature. Previously, pleural fluid cytology demonstrated presence of spheroid like cell aggregates with coiled cords of cells, small papillae with a central core and at times tissue fragments with pseudoacinar formation (5). Cell aggregation behavior confers resistance to anoikis through cell-cell interaction, calcium dependent intercellular junctions (14). Spheroid aggregates from cell cultures on non-adherent surfaces are chemo-resistant through Src family kinase(s) activation that quenches caspase activity (15). 
Glycolysis modulation occurs with the end product pyruvic acid. 3-Bromo pyruvic acid (3BrPA) has been experimentally tested in MSTO biphasic MPM cells (9). 3BrPA treatment lowered viability of MSTO cells in a timeand dose-dependent manner, and in vivo combination with CPDD prolonged survival. Similar modulation of glycolysis with the accumulation of citrate regulates glucose catabolism by inhibiting the key step of activating glucose by phosphofructokinase. Citrate mediated lowering of glucose metabolism in MPM has been reported with citrate+CPDD. Citrate in media activated caspase 3 and 9 suggestive of mitochondrial involvement, citrate combined with CPDD demonstrated a significant reduction in viability as opposed to citrate or CPDD (8). Also, 2DG has been reported as a combination therapeutic with docetaxel in patients with solid tumors (breast, thyroid, lung, head and neck). A dose of 63 $\mathrm{mg} / \mathrm{kg}$ was evaluated to be safe, with low adverse events and hence recommended for progression to phase II trial (13).

In conclusion our results showed that first-line chemotherapeutics in combination with 2-DG in MPM enhances the reduction of $2 \mathrm{D}$ cell proliferation, that is pertinent to tumor growth and spread in the thoracic wall and lung parenchyma. On the other hand, 3D spheroid experiments showed that addition of 2-DG to first-line chemotherapeutics does not further decrease the growth of spheroids implying that it would not influence the development of new tumor foci within the pleural cavity. Further experiments are required to determine the potential of 2-DG in negating MPM tumor growth and progression.

\section{Conflicts of Interest}

The Authors declare no conflicts of interest regarding this study.

\section{Authors' Contributions}

Irini Gerogianni, Rajesh M. Jagirdar, Chrissi Hatzoglou, and Sotirios G. Zarogiannis designed the study, analyzed the data, prepared the figures, and wrote the draft manuscript; Eleanna Pitaraki, Olympia Kouliou, Lydia Giannakou, Stefanos Giannopoulos and Eleftherios Papazoglou performed the experiments and revised the manuscript; Irene Gerogianni, Konstantinos I. Gourgoulianis and Sotirios G. Zarogiannis oversaw the study and revised the manuscript.

\section{Acknowledgements}

This work was supported by a Hellenic Thoracic Society Research Award 2017, awarded to Dr. Irini Gerogianni.

\section{References}

1 Kindler HL, Ismaila N, Armato III SG, Bueno R, Hesdorffer M, Jahan T, Jones CM, Miettinen M, Pass H, Rimner A, Rusch V, Sterman D, Thomas A and Hassan R: Treatment of malignant pleural mesothelioma: American Society of Clinical Oncology
Clinical Practice Guideline. J Clin Oncol 36(13): 1343-1373, 2018. PMID: 29346042. DOI: 10.1200/JCO.2017.76.6394.

2 Katzman D and Sterman DH: Updates in the diagnosis and treatment of malignant pleural mesothelioma. Curr Opin Pulm Med 24(4): 319-326, 2018. PMID: 29553973. DOI: 10.1097/ MCP.0000000000000489

3 Bibby AC, Tsim S, Kanellakis N, Ball H, Talbot DC, Blyth KG, Maskell NA and Psallidas I: Malignant pleural mesothelioma: an update on investigation, diagnosis and treatment. Eur Respir Rev 25(142): 472-486, 2016. PMID: 27903668. DOI: 10.1183/ 16000617.0063-2016

4 Finn RS, Brims FJH, Gandhi A, Olsen N, Musk AW, Maskell NA and Lee YCG: Postmortem findings of malignant pleural mesothelioma: a two-center study of 318 patients. Chest $142(5)$ : 1267-1273, 2012. PMID: 22576637. DOI: 10.1378/chest.113204

5 Michael CW, King JA and Hester RB: Confocal laser scanning microscopy and three-dimensional reconstruction of cell clusters in serous fluids. Diagn Cytopathol 17(4): 272-279, 1997. PMID: 9316783.

6 Cortes-Dericks L, Carboni GL, Schmid RA and Karoubi G: Putative cancer stem cells in malignant pleural mesothelioma show resistance to cisplatin and pemetrexed. Int J Oncol 37(2): 437-444, 2010. PMID: 20596671.

7 Hoshiba T and Tanaka M: Decellularized matrices as in vitro models of extracellular matrix in tumor tissues at different malignant levels: Mechanism of 5-fluorouracil resistance in colorectal tumor cells. Biochim Biophys Acta 1863(11): 27492757, 2016. PMID: 27558478. DOI: 10.1016/j.bbamcr. 2016.08.009

8 Zhang X, Varin E, Allouche S, Lu Y, Poulain L and Icard P: Effect of citrate on malignant pleural mesothelioma cells: a synergistic effect with cisplatin. Anticancer Res 29(4): 12491254, 2009. PMID: 19414371.

9 Zhang X, Varin E, Briand M, Allouche S, Heutte N, Schwartz L, Poulain L and Icard P: Novel therapy for malignant pleural mesothelioma based on anti-energetic effect: an experimental study using 3-Bromopyruvate on nude mice. Anticancer Res 29(4): 1443-1448, 2009. PMID: 19414400.

10 Jagirdar RM, Apostolidou E, Molyvdas PA, Gourgoulianis KI, Hatzoglou C and Zarogiannis SG: Influence of AQP1 on cell adhesion, migration, and tumor sphere formation in malignant pleural mesothelioma is substratum- and histological-type dependent. Am J Physiol Lung Cell Mol Physiol 310(6): L489495, 2016. PMID: 26773069. DOI: 10.1152/ajplung.00410.2015

11 Mi YJ, Geng GJ, Zou ZZ, Gao J, Luo XY, Liu Y, Li N, Li CL, Chen YQ, Yu XY and Jiang J: Dihydroartemisinin inhibits glucose uptake and cooperates with glycolysis inhibitor to induce apoptosis in non-small cell lung carcinoma cells. PLoS One 10(3): e0120426, 2015. PMID: 25799586. DOI: 10.1371/ journal.pone.0120426

12 Wang SY, Wei YH, Shieh DB, Lin LL, Cheng SP, Wang PW and Chuang JH: 2-Deoxy-d-glucose can complement doxorubicin and sorafenib to suppress the growth of papillary thyroid carcinoma cells. PLoS One 10(7): e0130959, 2015. PMID: 26134286. DOI: 10.1371/journal.pone.0130959

13 Raez LE, Papadopoulos K, Ricart AD, Chiorean EG, Dipaola RS, Stein MN, Rocha Lima CM, Schlesselman JJ, Tolba K, Langmuir VK, Kroll S, Jung DT, Kurtoglu M, Rosenblatt J and Lampidis TJ: A phase I dose-escalation trial of 2-deoxy-D- 
glucose alone or combined with docetaxel in patients with advanced solid tumors. Cancer Chemother Pharmacol 71(2): 523-530, 2012. PMID: 23228990. DOI: 10.1007/s00280-0122045-1

14 Daubriac J, Fleury-Feith J, Kheuang L, Galipon J, Saint-Albin A, Renier A, Giovannini M, Galateau-Sallé F and Jaurand MC: Malignant pleural mesothelioma cells resist anoikis as quiescent pluricellular aggregates. Cell Death Differ 16(8): 1146-1155, 2009. PMID: 19343038 . DOI: $10.1038 / \mathrm{cdd} .2009 .32$
15 Eguchi R, Fujita Y, Tabata C, Ogawa H, Wakabayashi I, Nakano T and Fujimori Y: Inhibition of Src family kinases overcomes anoikis resistance induced by spheroid formation and facilitates cisplatininduced apoptosis in human mesothelioma cells. Oncol Rep 34(5): 2305-2310, 2015. PMID: 26323315. DOI: 10.3892/ or.2015.4200

Received April 16, 2019

Revised June 9, 2019

Accepted June 10, 2019 